\title{
Corrigendum to "Spread of TEM, VIM, SHV, and CTX-M $\beta$-Lactamases in Imipenem-Resistant Gram-Negative Bacilli Isolated from Egyptian Hospitals"
}

\author{
El sayed Hamdy Mohammed, ${ }^{1}$ Ahmed Elsadek Fakhr, ${ }^{2}$ Hanan Mohammed El sayed, ${ }^{2}$ \\ Said abd Elmohsen Al Johery, ${ }^{2}$ Wesam Abdel Ghani Hassanein, ${ }^{1}$ \\ and Ahmed Mansour Alzohairy ${ }^{3}$ \\ ${ }^{1}$ Department of Botany \& Microbiology, Faculty of Science, Zagazig University, Zagazig 44511, Egypt \\ ${ }^{2}$ Department of Medical Microbiology \& Immunology, Faculty of Medicine, Zagazig University, Zagazig 44511, Egypt \\ ${ }^{3}$ Department of Genetics, Faculty of Agriculture, Zagazig University, Zagazig 44511, Egypt \\ Correspondence should be addressed to Ahmed Elsadek Fakhr; ahmed_fakhr@yahoo.com
}

Received 14 September 2016; Accepted 19 September 2016; Published 25 September 2017

Copyright (C) 2017 El sayed Hamdy Mohammed et al. This is an open access article distributed under the Creative Commons Attribution License, which permits unrestricted use, distribution, and reproduction in any medium, provided the original work is properly cited.

In the article titled "Spread of TEM, VIM, SHV, and CTX-M $\beta$-Lactamases in Imipenem-Resistant Gram-Negative Bacilli Isolated from Egyptian Hospitals" [1], Dr. Ahmed Mansour Alzohairy was missing from the authors' list. The corrected authors' list is shown above. In addition, the Acknowledgments section should be corrected as follows.

\section{Acknowledgments}

The authors would like to express their sincere thanks and deepest gratitude to the Microbiology \& Immunology Department, Zagazig Scientific and Medical Research Lab, Faculty of Medicine, Zagazig University, Egypt, in which the work was achieved.

\section{References}

[1] E. S. H. Mohammed, A. Elsadek Fakhr, H. M. El Sayed, S. A. E. Al Johery, and W. A. G. Hassanein, "Spread of TEM, VIM, SHV, and CTX-M $\beta$-lactamases in imipenem-resistant gramnegative bacilli isolated from Egyptian hospitals," International Journal of Microbiology, vol. 2016, Article ID 8382605, 15 pages, 2016. 

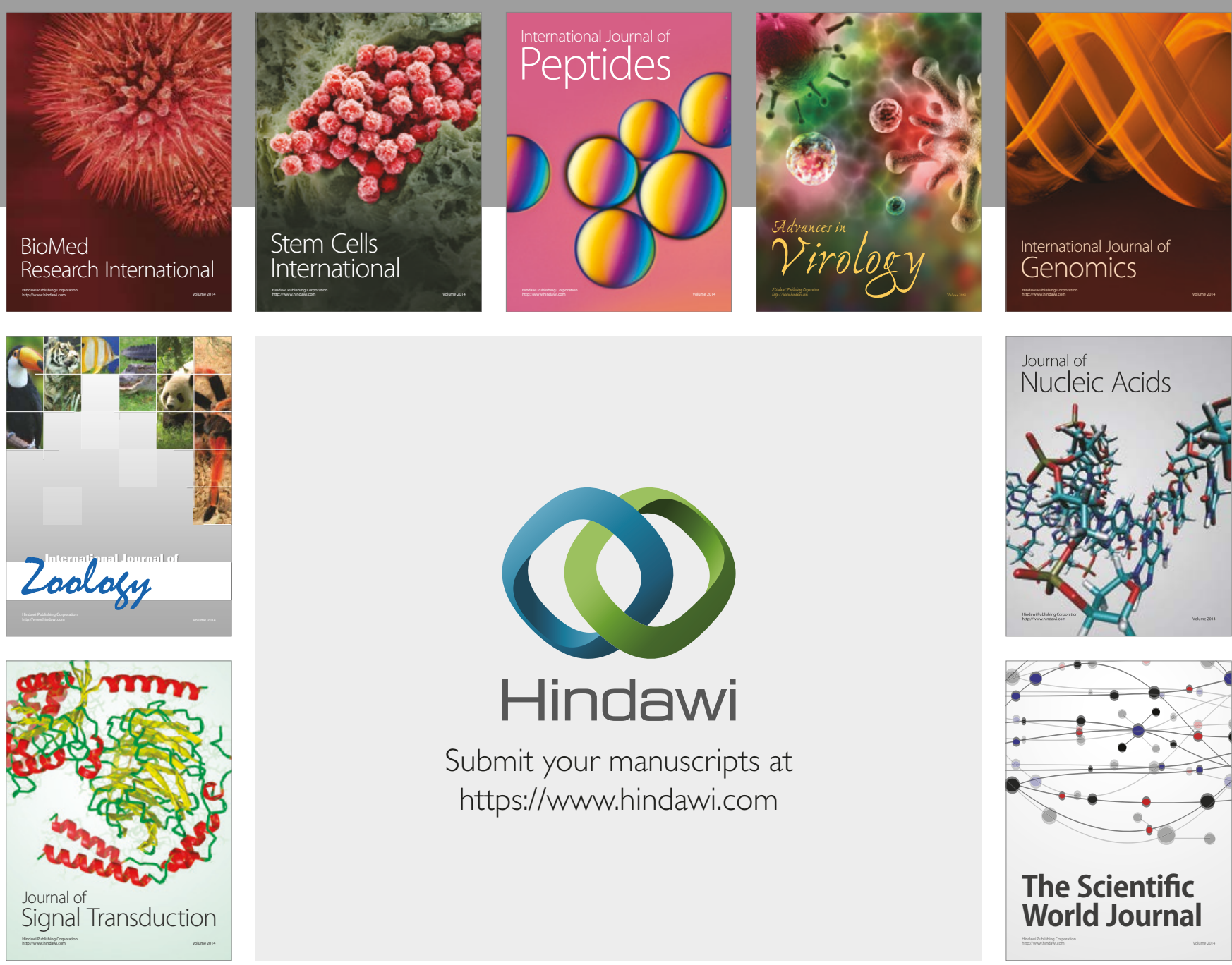

Submit your manuscripts at

https://www.hindawi.com
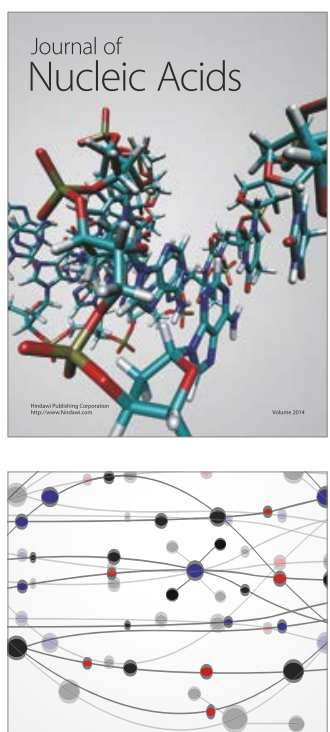

The Scientific World Journal

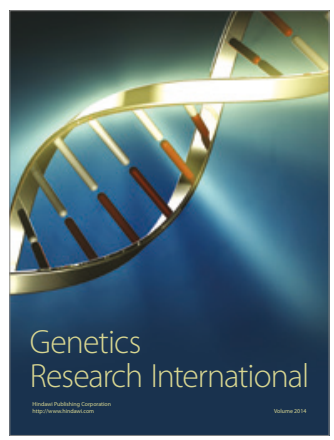

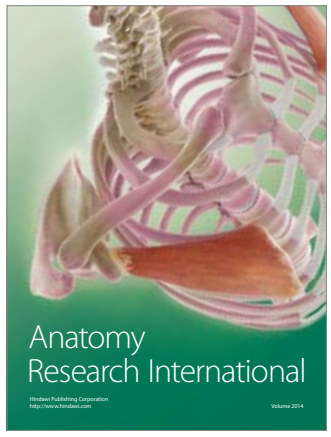

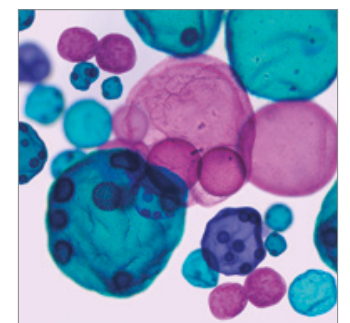

International Journal of Microbiology
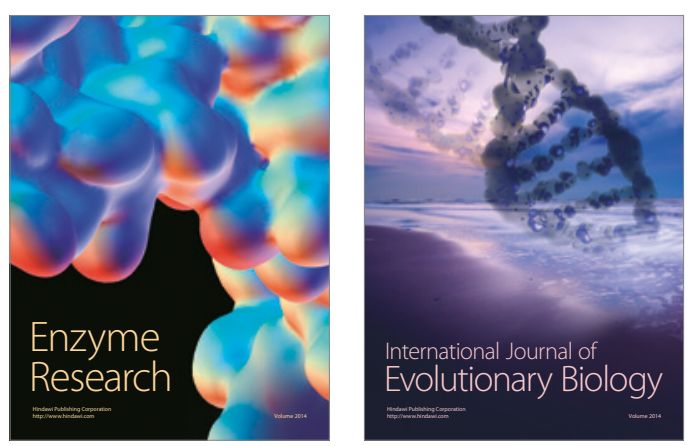
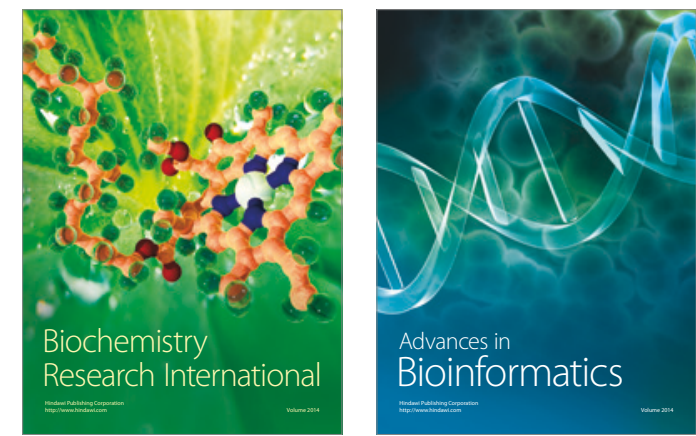

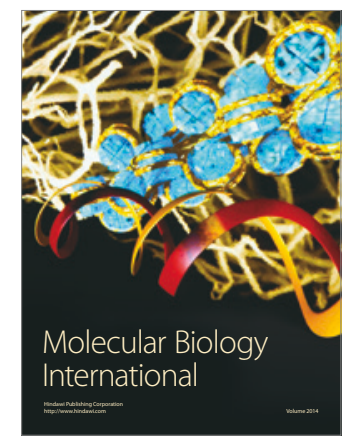

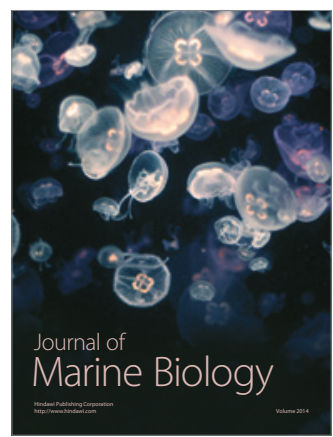

